\section{Uma revisão sobre os conceitos de acesso e utilização de serviços de saúde}

\author{
A review of concepts in health services \\ access and utilization
}

Claudia Travassos 1

Mônica Martins 2

\footnotetext{
1 Centro de Informação Científica e Tecnológica, Fundação Oswaldo Cruz, Rio de Janeiro, Brasil.

2 Escola Nacional de Saúde Pública, Fundação Oswaldo Cruz, Rio de Janeiro, Brasil.

Correspondência C. Travassos Departamento de Informações em Saúde, Centro de Informação Científica e Tecnológica, Fundação Oswaldo Cruz. Av. Brasil 4365, Rio de Janeiro, $R J$ 21045-900, Brasil. claudia@cict.fiocruz.br
}

\begin{abstract}
The purposes of this article are to review the concepts of health services access and utilization and to analyze how these concepts interrelate. Access is a complex concept (often used inaccurately) which changes over time and according to the context. Health services utilization is at the core of health systems functioning. Despite some disagreement, according to this review the prevailing perspective is that access is related to characteristics of services supply. Health care services utilization can be applied as a measure of access, but use of services depends on other factors. Individual and contextual factors influence the use of services. The article shows that the concept of access is becoming more comprehensive and is changing its focus from entry into the health system to outcome of care. Access is valued in relation to its impact on health and depends on the effectiveness of care delivered. As an outcome measure, access becomes multidimensional and difficult to operationalize. Finally, the article discusses how health determinants differ from those of health services utilization, which impacts directly on illness, but only indirectly on health.
\end{abstract}

Health Services; Utilization; Health Services Acessibility
A utilização dos serviços de saúde representa o centro do funcionamento dos sistemas de saúde. O conceito de uso compreende todo contato direto - consultas médicas, hospitalizações ou indireto - realização de exames preventivos e diagnósticos - com os serviços de saúde. O processo de utilização dos serviços de saúde é resultante da interação do comportamento do indivíduo que procura cuidados e do profissional que o conduz dentro do sistema de saúde. O comportamento do indivíduo é geralmente responsável pelo primeiro contato com os serviços de saúde, e os profissionais de saúde são responsáveis pelos contatos subseqüentes. Os profissionais, em grande parte, definem o tipo e a intensidade de recursos consumidos para resolver os problemas de saúde dos pacientes.

Em linhas gerais, os determinantes da utilização dos serviços de saúde podem ser descritos como aqueles fatores relacionados: (a) à necessidade de saúde - morbidade, gravidade e urgência da doença; (b) aos usuários - características demográficas (idade e sexo), geográficas (região), sócio-econômicas (renda, educação), culturais (religião) e psíquicas; (c) aos prestadores de serviços - características demográficas (idade e sexo), tempo de graduação, especialidade, características psíquicas, experiência profissional, tipo de prática, forma de pagamento; (d) à organização - recursos disponíveis, características da oferta (disponibilidade de médicos, hospitais, ambulatórios), modo 
de remuneração, acesso geográfico e social; (e) à política - tipo de sistema de saúde, financiamento, tipo de seguro de saúde, quantidade, tipo de distribuição dos recursos, legislação e regulamentação profissional e do sistema 1. A influência de cada um dos fatores determinantes do uso dos serviços de saúde varia em função do tipo de serviço (ambulatório, hospital, assistência domiciliar) e da proposta assistencial (cuidados preventivos ou curativos ou de reabilitação).

Acesso é um conceito complexo, muitas vezes empregado de forma imprecisa, e pouco claro na sua relação com o uso de serviços de saúde. É um conceito que varia entre autores e que muda ao longo de tempo e de acordo com o contexto. A terminologia empregada também é variável. Alguns autores, como Donabedian 2, empregam o substantivo acessibilidade - caráter ou qualidade do que é acessível 3 -, enquanto outros preferem o substantivo acesso - ato de ingressar, entrada 3 - ou ambos os termos para indicar o grau de facilidade com que as pessoas obtêm cuidados de saúde 4 .

Autores também variam em relação ao enfoque do conceito: uns centram-no nas características dos indivíduos; outros focam-no nas características da oferta; alguns em ambas as características ou na relação entre os indivíduos e os serviços (oferta). Há também discordâncias sobre se a avaliação de acesso deve concentrar-se nos resultados ou objetivos finais (goals) dos sistemas de saúde ou na relação entre os vários elementos que compõem o sistema para atingir esses objetivos 5 .

Neste artigo, pretende-se rever os conceitos de acesso e utilização de serviços de saúde, analisar o que os distingue e, ao mesmo tempo, identificar os pontos de articulação existentes entre ambos.

Em uma de suas primeiras publicações, Donabedian 2 definiu acessibilidade como um dos aspectos da oferta de serviços relativo à capacidade de produzir serviços e de responder às necessidades de saúde de uma determinada população. Acessibilidade, neste caso, é mais abrangente do que a mera disponibilidade de recursos em um determinado momento e lugar. Refere-se às características dos serviços e dos recursos de saúde que facilitam ou limitam seu uso por potenciais usuários. A acessibilidade corresponde a características dos serviços que assumem significado quando analisadas à luz do impacto que exercem na capacidade da população de usá-los. A acessibilidade é, assim, fator da oferta importante para explicar as variações no uso de serviços de saúde de grupos populacionais, e representa uma dimensão relevante nos estudos sobre a eqüidade nos sistemas de saúde.

Donabedian 2 distingue duas dimensões da acessibilidade: a sócio-organizacional e a geográfica e indica que essas dimensões se interrelacionam. Acessibilidade sócio-organizacional: inclui todas as características da oferta de serviços, exceto os aspectos geográficos, que obstruem ou aumentam a capacidade das pessoas no uso de serviços. Por exemplo: políticas formais ou informais que selecionam os pacientes em função de sua condição social, situação econômica ou diagnóstico. Acessibilidade geográfica: relaciona-se à fricção do espaço que pode ser medida pela distância linear, distância e tempo de locomoção, custo da viagem, entre outros. Apesar de atributos dos indivíduos (sociais, culturais, econômicos e psicológicos) não fazerem parte do conceito de acessibilidade de Donabedian, a relação destes com o uso de serviços é mediada pela acessibilidade, isto é, a acessibilidade expressa as características da oferta que intervêm na relação entre características dos indivíduos e o uso de serviços.

Donabedian 2, por um lado, delimita o escopo do seu conceito de acessibilidade ao excluir deste as etapas de percepção de problemas de saúde (necessidades) e o processo de tomada de decisão na procura de serviços pelos indivíduos. Por outro, avança na abrangência do conceito de acesso para além da entrada nos serviços, pois, para ele, acessibilidade indica também o grau de (des)ajuste entre as necessidades dos pacientes e os serviços e recursos utilizados. Acessibilidade não se restringe apenas ao uso ou não de serviços de saúde, mas inclui a adequação dos profissionais e dos recursos tecnológicos utilizados às necessidades de saúde dos pacientes.

Andersen ${ }^{6}$ prioriza o termo acesso. Em uma das versões iniciais do seu clássico modelo de utilização de serviços de saúde 7, acesso é apresentado como um dos elementos dos sistemas de saúde, dentre aqueles ligados à organização dos serviços, que se refere à entrada no serviço de saúde e à continuidade do tratamento. Abrange, nesse caso, a entrada nos serviços e o recebimento de cuidados subseqüentes.

Nesse modelo, a influência do acesso no uso de serviços de saúde é mediada por fatores individuais, definidos como: fatores predispo- 
nentes (fatores que existem previamente ao surgimento do problema de saúde e que afetam a predisposição das pessoas para usar serviços de saúde). Um bom exemplo é o gênero, já que as mulheres tendem a mostrar maior predisposição para o uso de serviços de saúde do que os homens 8,9; fatores capacitantes (os meios disponíveis às pessoas para obterem cuidados de saúde); e necessidades de saúde (condições de saúde percebidas pelas pessoas ou diagnosticadas por profissionais de saúde). Em concordância com Donabedian ${ }^{2}$, acesso é uma característica da oferta de serviços importante para explicação do padrão de utilização de serviços de saúde.

Em revisões posteriores do modelo de utilização de serviços, Andersen 6 amplia e clareia seu entendimento sobre o conceito de acesso, que passa explicitamente a incorporar a etapa de utilização de serviços de saúde. O conceito torna-se multidimensional, composto por dois elementos: "acesso potencial” e "acesso realizado". Acesso potencial caracteriza-se pela presença no âmbito dos indivíduos de fatores capacitantes do uso de serviços, enquanto acesso realizado representa a utilização de fato desses serviços e é influenciado por fatores outros além dos que explicam o acesso potencial.

$O$ conceito de acesso potencial incorpora os fatores individuais que limitam ou ampliam a capacidade de uso (fatores capacitantes), que representam apenas um subconjunto dos fatores que explicam o acesso realizado (uso), já que estes incluem também os fatores predisponentes, as necessidades de saúde, além de fatores contextuais.

Neste modelo, estabelece-se uma hierarquia na qual fatores contextuais, que são aqueles relacionados às políticas de saúde e à oferta de serviços, intervêm no uso de forma direta e indireta, por intermédio dos fatores individuais 6. Importante destacar, para fins de formulação de políticas, que elementos próprios do sistema de saúde (oferta) são passíveis de mudança mediante intervenção governamental ou institucional, enquanto apenas algumas das características dos indivíduos são passíveis de mudança por essas ações 5 .

Nas ultimas revisões, Andersen 6 busca incluir explicitamente no modelo os efeitos dinâmicos e recursivos do uso de serviços na saúde. Introduz os conceitos de "acesso efetivo" e de "acesso eficiente”. O primeiro resulta do uso de serviços que melhora as condições de saúde ou a satisfação das pessoas com os serviços; o segundo refere-se ao grau de mudança na saúde ou na satisfação em relação ao volume de serviços de saúde consumidos. Amplia o conceito de acesso realizado (uso) que passa a incluir os seus efeitos na saúde e na satisfação das pessoas. O resultado do acesso potencial pode ser medido pelo uso de serviços (acesso realizado) e o do acesso realizado (uso) pelo acesso efetivo e eficiente.

Entretanto, é importante lembrar que tanto o acesso realizado (uso) não se explica pelos determinantes do acesso potencial, como o seu impacto na saúde e na satisfação (acesso efetivo) não se explica apenas pelos determinantes do uso de serviços. Como apontado anteriormente, o uso de serviços depende de fatores predisponentes, das necessidades de saúde e de fatores contextuais, e o uso efetivo e eficiente depende dos fatores individuais e de fatores internos aos serviços de saúde que interferem na qualidade dos cuidados prestados.

Por fim, para Andersen 6, a avaliação da eqüidade no acesso é inferida valendo-se da presença de fatores individuais capacitantes na explicação do uso de serviços de saúde. Para este autor, a presença de outros preditores do uso, além da necessidade de saúde e dos fatores demográficas (predisponentes), denota uma situação de iniqüidade. Aponta também que a avaliação do acesso deve ser feita separadamente, segundo os tipos de cuidado (prevenção, cura e reabilitação), tipos de serviços (hospital e ambulatório) e tipos de problemas de saúde (atenção primária, especializada e de alta complexidade), pois expressam situações distintas com impacto diferenciado no acesso.

Penchansky \& Thomas 10 utilizam o termo acesso e centram esse conceito no grau de ajuste entre clientes e o sistema de saúde, numa interpretação da idéia desenvolvida por Donabedian 2. Diferem, no entanto, deste autor ao ampliarem o conceito para incluir outros atributos que são tomados não com base na oferta, mas sim na relação entre a oferta e os indivíduos. Identificam várias dimensões que compõem o conceito de acesso: disponibilidade (volume e tipo) de serviços em relação às necessidades; acessibilidade - tomada aqui como uma dimensão do acesso -, caracterizada pela adequação entre a distribuição geográfica dos serviços e dos pacientes; acolhimento (accomodation), que representa a relação entre a forma como os serviços organizam-se para receber os clientes e a capacidade dos clientes para se adaptar a essa organização; capacidade de compra, definida pela relação entre formas de financiamento dos serviços e a possibilidade das pessoas de pagarem por esses serviços; e aceitabilidade, que representa as atitudes das pessoas e dos profissionais de saúde em relação às características e práticas de cada um. 
A proposta de Penchansky \& Thomas de ampliação da abrangência do conceito de acesso com a incorporação de distintas dimensões não é seguida por muitos autores que, tal como Frenk 11, preferem manter o conceito em um domínio mais restrito. Donabedian 12, prefere denominar de qualidade - eficácia, efetividade, eficiência, otimização, aceitabilidade, legitimidade e eqüidade - o domínio mais amplo do desempenho dos sistemas de saúde.

Frenk 11 também desenvolve o conceito de acessibilidade fundamentando-se na proposta de Donabedian. Dentre as contribuições desse autor, estão a sistematização do fluxo de eventos entre a necessidade e a obtenção dos cuidados necessários (necessidades de saúde $\Rightarrow$ desejo de obter cuidados de saúde $\Rightarrow$ procura $\Rightarrow$ entrada nos serviços $\Rightarrow$ continuidade dos cuidados) e a explícita limitação do âmbito da acessibilidade às etapas de procura e entrada nos serviços. Desta forma, os determinantes das necessidades e do desejo de procurar cuidados de saúde, que podem ser incluídos no que Andersen denomina de fatores predisponentes, assim como aqueles que determinam a continuidade dos cuidados, permanecem fora do domínio da acessibilidade. Os primeiros compõem os modelos explicativos do uso e o último deve ser tratado no domínio da "continuidade", que depende mais de fatores associados à oferta do que aos indivíduos.

Frenk desenvolve o conceito de acessibilidade pela idéia de complementaridade entre características da oferta e da população. Para esse autor, acessibilidade é a relação funcional entre um conjunto de obstáculos para procurar e obter cuidados ("resistência") e as correspondentes capacidades da população para superar tais obstáculos ("poder de utilização”). A resistência inclui aqueles impedimentos que não se referem à mera disponibilidade de serviços, condição sine qua non do uso. Esses obstáculos (resistência) são classificados como ecológicos, financeiros e organizacionais. De forma correspondente, o poder da população é discriminado em poder de tempo e transporte, poder financeiro e poder de lidar com a organização. Esse modelo assume que, dados um lugar e um tempo, vários ajustes entre o poder de uso da população e as resistências da oferta são possíveis para um mesmo nível de acessibilidade. $\mathrm{O}$ central nessa abordagem é que nenhum desses dois componentes - resistência e poder de utilização da população - define o grau de acessibilidade, mas sim a relação entre eles. Por exemplo, o impacto na acessibilidade do aumento no preço dos serviços de saúde somente pode ser avaliado quando comparado com o nível de renda da população. Desta forma, sua formulação não aponta para um modelo organizacional único, mas sim para várias composições de oferta de serviços organizadas de acordo com o poder de utilização dos diferentes grupos populacionais (sociais).

No início da década de 90, o Comitê para o Monitoramento do Acesso aos Serviços de Saúde do Institute of Medicine (IOM) dos Estados Unidos propõe que acesso seja definido como o uso de serviços de saúde em tempo adequado para obtenção do melhor resultado possível 13. Nessa definição, o eixo do conceito é deslocado dos elementos que o compõem para seus resultados e acesso passa a ser sinônimo de uso, além de ser incorporada ao conceito a dimensão temporal. Esta dimensão está presente em outros autores 14,15 que consideram acesso (ótimo) como a provisão do cuidado adequado, no momento adequado e no local adequado. Acesso, neste caso, não abrange qualquer uso; limita-se ao uso qualificado, a saber, aquele que ocorre no momento adequado ao atendimento do problema de saúde do paciente, utilizando recursos corretos e executado da forma correta. Este ajuste entre o problema de saúde do paciente e os recursos e a forma como são empregados já havia sido indicado por Donabedian 16 em seu conceito de acessibilidade, como apontado anteriormente.

Na abordagem do IOM, a importância do acesso centra-se no uso de procedimentos de saúde com potencial para alterar positivamente o estado de saúde das pessoas. $\mathrm{O}$ acesso refere-se ao uso, no momento adequado, de serviços/tecnologias de reconhecida eficácia. Interessa saber se oportunidades de bons resultados (alteração positiva nas condições de saúde) estão sendo perdidas por problemas de “acesso". Contudo, uma importante limitação à utilidade desse conceito é que a eficácia de grande parte dos procedimentos preventivos e terapêuticos é desconhecida 17. A outra limitação é que mudanças positivas no estado de saúde dependem não apenas do acesso a procedimentos de saúde de reconhecida eficácia, porém, conforme já indicado, da adequação na realização do procedimento (qualidade técnica), o que torna a operacionalização do conceito bastante difícil.

O conceito de acesso do IOM é semelhante ao que Andersen 6 denomina de "acesso efetivo”. No caso do IOM, este conceito assume um caráter normativo, pois incorpora apenas um subconjunto de intervenções, aquele considerado, com base em critérios pré-estabelecidos, como capaz de produzir impacto positivo nas condições de saúde da população. Ao mesmo 
tempo, como conceito de resultado, não leva em conta os fatores do sistema de saúde que explicam as variações nos padrões de uso dessas intervenções em diferentes grupos populacionais. O enfoque da avaliação de acesso volta-se para diagnosticar a existência de variações no acesso (uso) a procedimentos específicos e não para explicá-los. Para autores como Gold 18, como as dimensões do desempenho dos sistemas de saúde - acesso, custo, efetividade e satisfação - tornam-se cada vez mais inter-relacionadas, justifica-se o deslocamento do eixo do acesso para os resultados dos cuidados.

Numa abordagem semelhante, a Organização Mundial da Saúde propôs para discussão um novo indicador de avaliação do desempenho dos sistemas de saúde que denominou de "cobertura efetiva". Cobertura efetiva foi definida como a proporção da população que necessita de um determinado procedimento de saúde e que recebeu de forma efetiva este procedimento 19 . Tal como na definição de acesso do IOM, representa uma medida de resultado abrangente que mede tanto o acesso potencial, quanto o acesso realizado (uso) e o acesso efetivo (qualidade do cuidado). Apresenta limitações semelhantes às da medida do IOM, acrescidas de problemas no cálculo da cobertura, devido às dificuldades para identificação do numerador e denominador. Estes têm que considerar apenas aqueles indivíduos para os quais o procedimento em questão tem eficácia reconhecida e esta informação não é de fácil obtenção. Um outro problema é que, além da ocorrência de subutilização pela população que necessita, existe um percentual, dentre os pacientes que recebem o procedimento, que dele não necessitavam. Estes últimos não devem compor o denominador da cobertura efetiva, no entanto, para identificá-los, há que proceder a estudos específicos (http://www.rand. org/helath/suerveys/sf36item/questinnaire. html, acessado em 24/Mar/2004).

Com base na definição do IOM foram propostas várias medidas de acesso, agrupadas em cinco objetivos, que cobrem as fases da vida (Tabela 1). Entretanto, as medidas de acesso propostas são predominantemente representadas por indicadores tradicionais de cobertura - proporção da população que tem acesso potencial - ou por taxas de utilização de determinados serviços ou procedimentos. São tradicionais, pois, na sua maioria, não exprimem o resultado na saúde produzido pelo uso de serviços, de acordo com o sugerido pelo conceito.

Vários autores 18,20 assumem que acesso é um conceito contexto dependente. Gold 18, ainda que reconhecendo a importância do uso de medidas tradicionais de acesso (acesso potencial), destaca a necessidade do desenvolvimento desse conceito e de sua operacionalização para dar conta das recentes mudanças e da grande variação dos mercados em saúde nos Estados Unidos.

Contudo, variações contextuais devem afetar menos o conceito e mais a especificação dos

Tabela 1

Áreas assistenciais e respectivos indicadores de acesso. Comitê de Monitoramento do Acesso aos Serviços de Saúde, Instituto de Medicina, Estados Unidos.

\begin{tabular}{ll}
\hline Objetivos & Atividades e indicadores \\
\hline $\begin{array}{l}\text { Promoção de bons resultados no nascimento } \\
\text { Redução da incidência em crianças de }\end{array}$ & $\begin{array}{l}\text { Mortalidade infantil } \\
\text { Baixoso ao nascer } \\
\text { Incia de sífilis congênita }\end{array}$ \\
$\begin{array}{l}\text { Detecção e diagnóstico precoce de doenças } \\
\text { passíveis de tratamento }\end{array}$ & Vacinação \\
$\begin{array}{l}\text { Redução dos efeitos das doenças crônica } \\
\text { e prolongamento da vida }\end{array}$ & Rastreamento de câncer de mama e de colo de útero \\
$\begin{array}{l}\text { Uso de procedimentos eletivos de alto custo } \\
\text { Redução da morbidade e da dor através de tratamento } \\
\text { oportuno e adequado }\end{array}$ & $\begin{array}{l}\text { Internações por grupos específicos de diagnósticos } \\
\text { Uso de serviços odontológicos }\end{array}$
\end{tabular}

Fonte: Millman 13 . 
modelos explicativos de acesso e a sua operacionalização, de forma a ter em foco as características particulares de cada sistema de saúde. Por exemplo, cobertura de plano de saúde é um indicador tradicional de acesso nos Estados Unidos, e importante atualmente no Brasil, mas inexpressivo nos países europeus que dispõem de sistema de saúde com cobertura universal. $\mathrm{O}$ mesmo pode ser dito sobre os determinantes da escolha do plano de saúde, proposto por Gold 18, que não compõem os modelos explicativos do acesso em sistemas de saúde baseados em seguro público de saúde. Variações nas coberturas a procedimentos específicos, nos tipos de prestadores de serviços credenciados, nos incentivos financeiros para o uso desses serviços, no emprego de outras medidas de racionamento do uso como o co-pagamento, na existência de porta de entrada, de regras para encaminhamento de pacientes, no emprego de protocolos de cuidado e no monitoramento de qualidade representam algumas dessas características que diferenciam os mercados de saúde.

Starfield 17 distingue acesso de acessibilidade. Acessibilidade refere-se a características da oferta e o acesso é a forma como as pessoas percebem a acessibilidade. Outros autores 21 também apontam que a forma como as pessoas percebem a disponibilidade de serviços afeta a decisão de procurá-los. Essa percepção é influenciada pela experiência passada com os serviços de saúde. Goddard \& Smith 20 destacam o fato de que a disponibilidade de serviços pode também não ser de conhecimento de todos e que diferentes grupos populacionais variam no grau de informação que possuem sobre os serviços a eles disponíveis. Desse modo, a experiência com os serviços e as informações que deles dispõem influenciam a forma como as pessoas percebem as dificuldades/facilidades para obterem os serviços de saúde de que necessitam e, portanto, o acesso aos mesmos.

\section{Utilização de serviços}

As primeiras tentativas de desenvolvimento de modelos teóricos sobre a utilização dos serviços de saúde datam das décadas de 50 e 60, destacando-se como precursores o modelo de crenças em saúde (health belief model), apresentado na década de 50, e o modelo de Andersen 6 de 1968. Esses modelos ganharam maior complexidade e evoluíram com base nos conceitos e relações neles esboçados.

Haddad 22 realizou uma extensa revisão bibliográfica sobre os modelos explicativos do processo de utilização dos serviços de saúde e classificou os modelos revistos como: (a) modelos centrados nas etapas de tomada de decisão e no comportamento individual; (b) modelos de interface, nos quais a utilização é produto da interação entre os indivíduos e os prestadores de serviço. Dentre os modelos existentes serão destacados neste artigo os seguintes: (a) o modelo de crenças em saúde 23; (b) os modelos de Andersen \& Newman 7 e de Aday \& Andersen 5; (c) o modelo de Dutton 24 e (d) o modelo de Evans \& Sttodart 25.

O modelo comportamental baseado nas crenças dos indivíduos foi desenhado nos anos 50 para explicar falhas na adesão aos programas de prevenção e detecção precoce de doenças. Posteriormente, esse modelo passou a ser aplicado na análise do comportamento dos indivíduos frente a sintomas e doenças e, sobretudo, para estudar a aderência à prescrição terapêutica 23 . Ele busca explicar o comportamento dos indivíduos em relação à saúde e à utilização dos serviços tomando por base as crenças, intenções e percepções dos riscos.

O comportamento dos indivíduos em relação à saúde depende se consideram suscetíveis a um determinado problema de saúde, se acreditam na gravidade das conseqüências deste problema e se acreditam que as ações de saúde disponíveis podem trazer-lhes benefícios 23. A suscetibilidade percebida refere-se à percepção subjetiva do risco de ter doença. A gravidade percebida refere-se aos sentimentos e preocupações com relação a uma doença e suas conseqüências na saúde (morte, dor ou incapacidade) e nas condições de vida (condições de trabalho, vida familiar e relações sociais). A probabilidade do indivíduo adotar uma "ação de saúde" é influenciada por três componentes: (a) a propensão à ação; (b) a avaliação das vantagens e dos inconvenientes de adotar esta ação; e (c) os estímulos internos e externos para adotá-la. O balanço entre benefícios e barreiras percebidas com relação à ação a ser adotada é descrito como uma análise inconsciente de custo-benefício em que indivíduos pesam os ganhos da ação contra a percepção de que esta ação pode ser custosa, perigosa, desagradável ou inconveniente. A ameaça notada e a percepção de que os benefícios são superiores às barreiras para ação são os elementos-chave para a adoção de uma determinada ação preventiva ou prescrição terapêutica 23 .

Dentre esses modelos de explicação da utilização de serviços de saúde existentes, aquele proposto por Andersen \& Newman 7 tem sido o mais aplicado tanto nos estudos de utilização, quanto nos estudos de acesso. Neste modelo, 
cuja origem é o trabalho de Andersen de 1968 , o uso de serviços é dependente de determinantes individuais agrupados nos fatores de predisposição, fatores capacitantes e necessidades de saúde. O modelo indica a existência de uma relação entre esses três fatores, de forma que os fatores predisponentes influenciam os capacitantes. As necessidades representam o determinante mais proximal da utilização dos serviços de saúde. Em sua evolução, passa a explicar a utilização como produto não exclusivo dos determinantes individuais, mas sim como fruto dos fatores individuais, do sistema de saúde e do contexto social, da interação entre esses fatores e da experiência passada de utilização dos serviços. Cumpre ressaltar que até recentemente os determinantes contextuais (determinantes da sociedade e do sistema de saúde) tinham sido operacionalizados nos estudos empíricos 26.

Vários autores apontam que fatores da oferta podem induzir demanda aos serviços de saúde 27,28,29,30. Tendo-se em conta a importância dos fatores ligados à oferta no uso dos serviços, ainda são poucos os modelos que incluem as características individuais do prestador/profissional de saúde como fator de explicação do uso; exceção é o modelo proposto por Dutton 24. Nele a utilização dos serviços de saúde é compreendida como produto da interação entre clientes, prestadores de cuidado de saúde (profissionais) e sistema de saúde. As características dos pacientes são compostas pelos determinantes individuais definidos por Andersen 6 . Nas características dos médicos são considerados os seguintes elementos: demográficos, formação, experiência e atitudes. O sistema de saúde é abordado nesse modelo por intermédio dos obstáculos estruturais que influenciam a utilização. Os principais são: obstáculos financeiros; temporais (tempo de espera e distância), organizacionais e aqueles ligados à prática médica.

Dutton distingue, no modelo, o uso de serviços cujo controle é preponderantemente determinado pelo paciente (exames preventivos e primeiro contato) daquele serviço cujo controle é determinado pelos médicos (consultas subseqüentes e tratamento prescrito). A utilização dos serviços controlada pelo paciente dependerá de suas características e das barreiras estruturais do sistema de saúde. Já o uso dos serviços controlado pelos médicos dependerá da necessidade de saúde e das características de tais profissionais. Por conseguinte, esse autor também destaca que os elementos determinantes de uma consulta de primeira vez são distintos daqueles que influenciam as con- sultas subseqüentes. Porém, a necessidade de saúde é um determinante importante tanto no uso controlado pelo pacientes como no controlado pelo médico.

Uma tentativa mais recente de pensar a inter-relação entre os elementos que explicam o uso dos serviços de saúde, sobretudo a relação entre saúde e uso de serviços de saúde, é o modelo proposto por Evans \& Stoddart 25, denominado "Modelo de Produção da Saúde". Destaca-se por descrever a relação causal recíproca entre doença e utilização, em que somente a doença, e não a saúde, é responsável direta pelo consumo de serviços de saúde. A saúde é tomada como fenômeno distinto da doença, que não se reduz à sua simples ausência. Essa abordagem vem ao encontro do conceito de saúde apresentado por Contandriopoulos 31, para o qual não existe um contínuo entre a saúde e a doença, isto é, do estado mais completo de saúde e bem-estar até a morte, passando pelos diferentes estágios das doenças e das perdas de capacidade funcional. O modelo explicita que os determinantes da saúde diferem dos determinantes do uso de serviços de saúde. A doença - fator diretamente associado ao uso - é um dos elementos, dentre outros, como o grau de prosperidade e bem-estar de uma sociedade, que determinam a saúde. A utilização de serviços impacta diretamente a doença, mas apenas indiretamente a saúde.

Concebido teoricamente para dar conta da complexidade dos fatores e dimensões que interferem no processo saúde, doença e uso dos serviços, esse modelo não foi empiricamente testado, necessitando ainda de maior especificação de seus elementos. Sua principal qualidade até o momento é sua robusteza teórica.

\section{Considerações finais}

Ao término desta revisão, percebe-se que, apesar das discordâncias na terminologia e na abrangência dos conceitos de utilização e acesso, podem ser traçadas algumas linhas de concordância entre os autores. Quanto à terminologia, a acessibilidade é preferida pelos autores que a descrevem como uma característica da oferta de serviços de saúde ou do ajuste entre a oferta e a população, seja esta uma característica geral 2,11, seja restrita à acessibilidade geográfica 10. Já os autores que optam pelo termo acesso, em geral, centram-no na entrada inicial dos serviços de saúde, podendo-se citar, como exemplo, o modelo de Andersen 6 . De qualquer forma, prevalece a idéia de que acesso é uma dimensão do desempenho dos sistemas de saú- 
de associada à oferta. Quanto à abrangência do conceito, observa-se uma tendência de ampliação do escopo, com deslocamento do seu eixo da entrada nos serviços para os resultados dos cuidados recebidos. Não obstante a tendência recente de empregar-se o conceito baseado em resultados, argumenta-se sobre a importância de manterem-se as distinções entre acesso e uso de serviços de saúde; acesso e continuidade do cuidado; e acesso de efetividade dos cuidados prestados. A principal razão é que cada um destes processos corresponde a um modelo explicativo distinto.

O uso de serviços pode ser uma medida de acesso, mas não se explica apenas por ele. A despeito de o acesso ser um importante determinante do uso, o uso efetivo dos serviços de saúde resulta de uma multiplicidade de fatores. Fatores individuais predisponentes, fatores contextuais e relativos à qualidade do cuidado influenciam o uso e a efetividade do cuidado. A continuidade também depende de situações distintas daquelas que definem a entrada no sistema, o que indica a importância de que seja analisada em suas particularidades.

\section{Resumo}

O objetivo deste artigo é rever os conceitos de acesso $e$ de utilização de serviços de saúde, identificando pontos de distinção e articulação existentes entre ambos. Acesso é um conceito complexo, geralmente empregado de forma imprecisa e que muda ao longo do tempo e de acordo com o contexto. A utilização dos serviços de saúde representa o centro do funcionamento dos sistemas de saúde. Apesar das divergências, predomina a visão de que o acesso relaciona-se a características da oferta de serviços. O uso de serviços é uma expressão do acesso, mas não se explica apenas por ele. Fatores individuais predisponentes e contextuais também influenciam o uso. Observa-se uma tendência de ampliação do escopo do conceito de acesso, com deslocamento do seu eixo da entrada nos serviços (uso) para os resultados dos cuidados recebidos. O acesso é visto pelo seu impacto na saúde e dependerá também da adequação do cuidado prestado. Finalmente, destacase que determinantes da saúde diferem daqueles do uso de serviços e que a utilização de serviços impacta diretamente a doença, mas apenas indiretamente a saúde.

Serviços de Saúde; Utilização; Acessibilidade aos Serviços de Saúde
A atual tendência de se deslocar o conceito de acesso para os resultados dos cuidados de saúde também apresenta limitações para sua avaliação, uma vez que requer medidas multidimensionais de difícil compreensão, operacionalização e, muitas vezes, de baixa validade.

Um outro aspecto importante destacado pelos modelos analisados é a distinção entre os modelos explicativos da saúde e do uso de serviços de saúde. Saúde é entendida como um fenômeno bem mais amplo que a doença e não se explica unicamente pelo uso de serviços de saúde. Desta forma, a saúde da população não resulta diretamente da ação dos sistemas de saúde.

Concluindo, considera-se que se deve priorizar o emprego de medidas que apreendam cada uma das etapas no processo de utilização de serviços, de forma a permitir a verificação do efeito dos múltiplos fatores explicativos das variações no uso de serviços, como destas nas condições de saúde das pessoas e desta forma orientar melhor a formulação de políticas para um melhor desempenho dos sistemas de saúde. Para tal é fundamental a construção de modelos teóricos que incorporem as particularidades de cada contexto a ser analisado.

\section{Colaboradores}

C. Travassos e M. Martins participaram de todas as etapas de levantamento e revisão bibliográfica, estruturação e redação do texto. 


\section{Referências}

1. Pineault R, Daveluy C. Les indicateurs d"tilisation des services de santé. In: Pineault R, Daveluy C, editors. La planification de la santé: concepts, méthodes et stratégies. Montréal: Agence d'Arc; 1986. p. 191-202.

2. Donabedian A. Aspects of medical care administration. Boston: Harvard University Press; 1973.

3. Houaiss A, Villar M. Minidicionário Houaiss da língua portuguesa. Rio de Janeiro: Objetiva; 2001.

4. Donabedian A. An introduction to quality assurance in health care. New York: Oxford University Press; 2003.

5. Aday LA, Andersen R. A framework for the study of access to medical care. Health Serv Res 1974; 9:208-20.

6. Andersen RM. Revisiting the behavioral model and access to medical care: does it matter? J Health Soc Behav 1995; 36:1-10.

7. Andersen RM, Newman JF. Societal and individual determinants of medical care utilization in the United States. Milbank Mem Fund Q 1973; 51: 95-124.

8. Hulka BS, Wheat JR. Patterns of utilization - the patient perspective. Med Care 1985; 23:438-60.

9. Pinheiro RS, Viacava F, Travassos C, Brito AS. Gênero, morbidade, acesso e utilização de serviços de saúde no Brasil. Ciênc Saúde Coletiva 2002; 7:687-707.

10. Penchansky DBA, Thomas JW. The concept of access - definition and relationship to consumer satisfaction. Med Care 1981; 19:127-40.

11. Frenk J. Conceptand measurement of accessibility. Salud Publica Mex 1985; 27:438-53.

12. Donabedian A. The seven pillars of quality. Arch Pathol Lab Med 1990; 114:1115-8.

13. Millman M. Access to health care in America. Washington DC: National Academy Press; 1993.

14. Rogers A, Flowers J, Pencheon D. Improving access needs a whole systems approach. BMJ 1999; 319:866-7.

15. Joint Commission on Accreditation of Healthcare Organizations. The measuring mandate - on the road to performance improvement in health care. Chicago: Joint Commission on Accreditation of Healthcare Organizations; 1993.

16. Donabedian A. The assessment of need. In: Donabedian A, editor. Aspects of medical care administration. Cambridge: Harvard University Press; 1973. p. 58-77.

17. Starfield B. Acessibilidade e primeiro contato: a "porta". In: Starfield B, organizador. Atenção primária - equilíbrio entre necessidades de saúde serviços e tecnologia. Brasília: Organização das Nações Unidas para a Educação, a Ciência e a Cultura/Ministério da Saúde; 2002. p. 207-45.

18. Gold M. Beyond coverage and supply: measuring access to healthcare in today's market. Health Serv Res 1998; 33:625-52.
19. World Health Organization. Background paper for the technical consultation on effective coverage of health systems. Geneva: World Health Organization; 2001.

20. Goddard M, Smith P. Equity of access to health care services: theory and evidence from the UK. Soc Sci Med 2001; 53:1149-62.

21. Carr-Hill RA, Hardman G, Martin S, Peacock S, Sheldon TA, Smith P. A formula for distributing NHS revenues based on small area use of hospital beds. York: Centre for Health Economics, University of York; 1994.

22. Haddad S. Utilization des services de santé en pays en développement [Tese de Doutorado]. Lyon: Université Claude Bernard; 1992.

23. Rosenstock IM. The health belief model: explaining health behavior through expectancies. In: Glanz K, Levis FM, Rimer BK, editors. Health behavior and health education: theory, research and practice. San Francisco: Jossey-Bass; 1990. p. 39-62.

24. Dutton D. Financial, organizational and professional factors affecting health care utilization. Soc Sci Med 1986; 23:721-35.

25. Evans RG, Stoddart GL. Producing health, consuming health care. In: Evans RG, Barer ML, Marmor TR, editors. Why are some people health and others not?: the determinants of health of populations. New York: Aldine DeGruyter; 1994. p. 27-64.

26. Phillips K, Morrison K, Andersen R, Aday L. Understanding the context of healthcare utilization: assessing environmental and provider-related variables in the behavioral model of utilization. Health Serv Res 1998; 33:571-96.

27. Wennberg J, Gittelsohn A. Small area variations in health care delivery. Science 1973; 182:1102-9.

28. McPherson K, Wennberg JE, Hovind OB, Clifford P. Small area variations in the use of common surgical procedures: an international comparation of New England, England and Norway. N Engl J Med 1982; 307:1310-4

29. Eisenberg JM. Variation in medical decision making. In: Eisenberg JM, editor. Doctors' decisions and the cost of medical care. Ann Arbor: Health Administration Press; 1986. p. 5-27.

30. Roos N, Roos L. Small area variations, practice style, and quality of care. In: Evans R, Barer M, Marmor T, editors. Why are some people healthy and others not? The determinants of health of populations. Hawthorne: Aldine DeGruyter; 1994. p. 231-52.

31. Contandriopoulos AP. La salud entre las ciencias de la vida y las ciencias sociales. Cuad Med Soc 1999; 77:19-33.

Recebido em 22/Jun/2004

Aprovado em 23/Ago/2004 